\title{
Amiodarone-Induced Thyrotoxicosis
}

Ricardo Fonseca, Catarina Roque, Carlos Bello, Francisco Sousa Santos, Ricardo Capitão, João Sequeira Duarte, Carlos Vasconcelos ENDOCRINOLOGY DEPARTMENT | HOSPITAL EGAS MONIZ (CHLO) | LISBON, PORTUGAL

\section{BACKGROUND}

Amiodarone is an antiarrhytmic drug mainly used in atrial fibrillation. Amiodarone-induced thyrotoxicosis (AIT) is less common than hypothyroidism, but it represents a true therapeutic challenge. AIT can be due iodine load (type 1) or a destructive thyroiditis (type 2 ).

\section{METHODS}

Retrospective study of patients with AIT, diagnosed between 2010 and 2015, in a central hospital. We reviewed medical records regarding age, gender, amiodarone therapy, hospitalizations, cardiovascular events, thyroid function and therapy. We used descriptive statistics, t-test for continuous variables and chi-squared distribution for categorical variables.

\section{RESULTS}

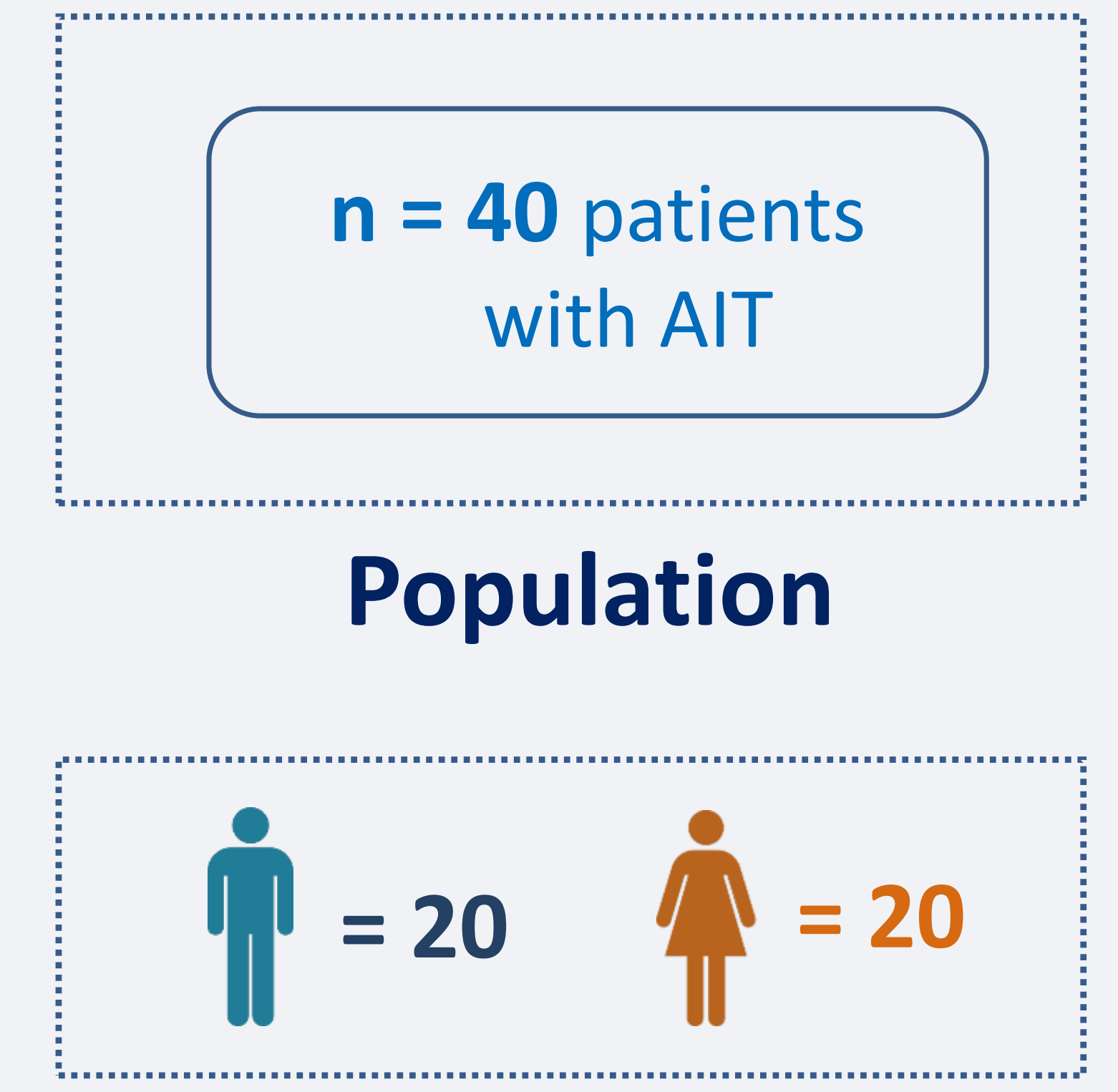

Gender

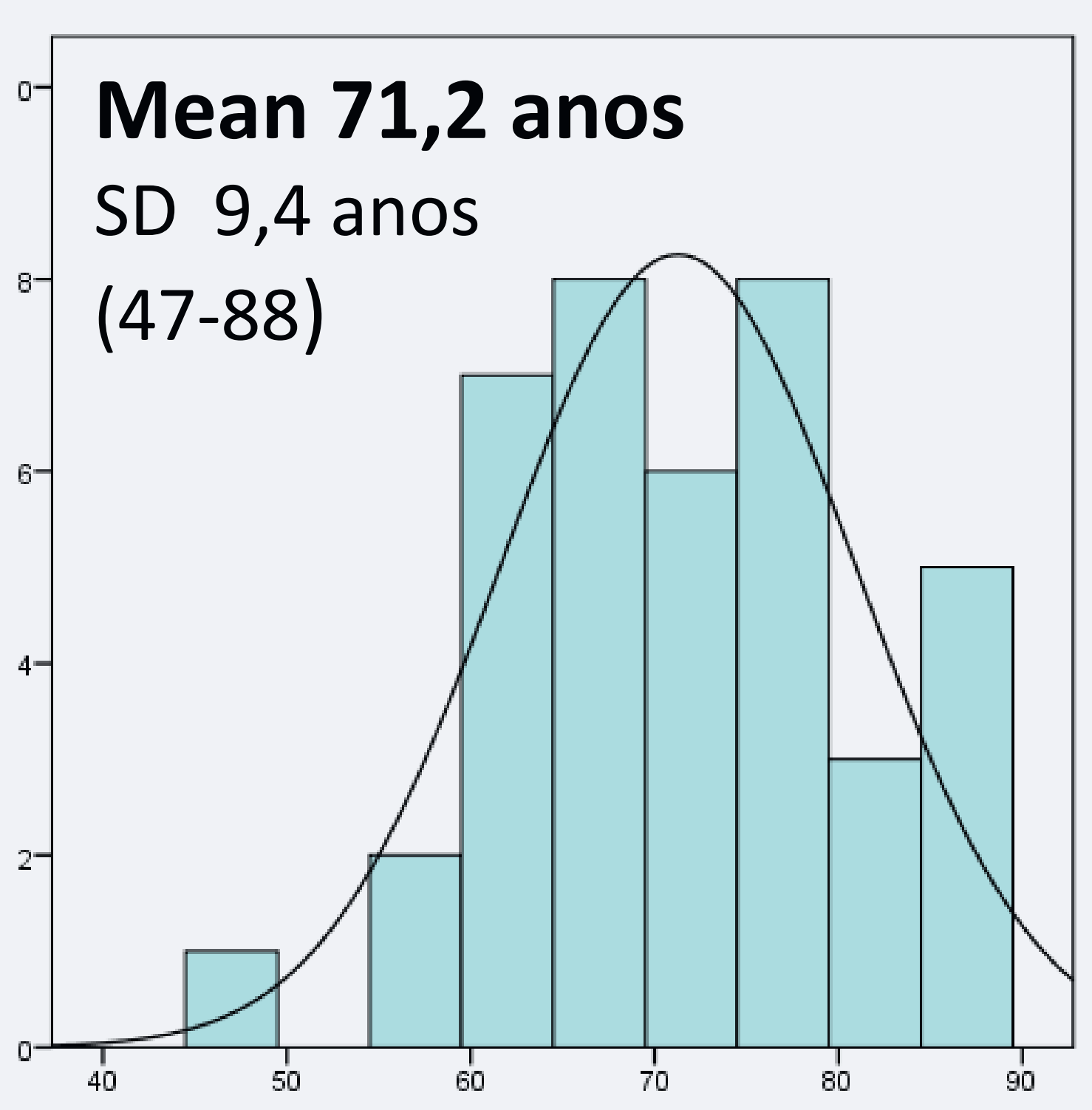

Age

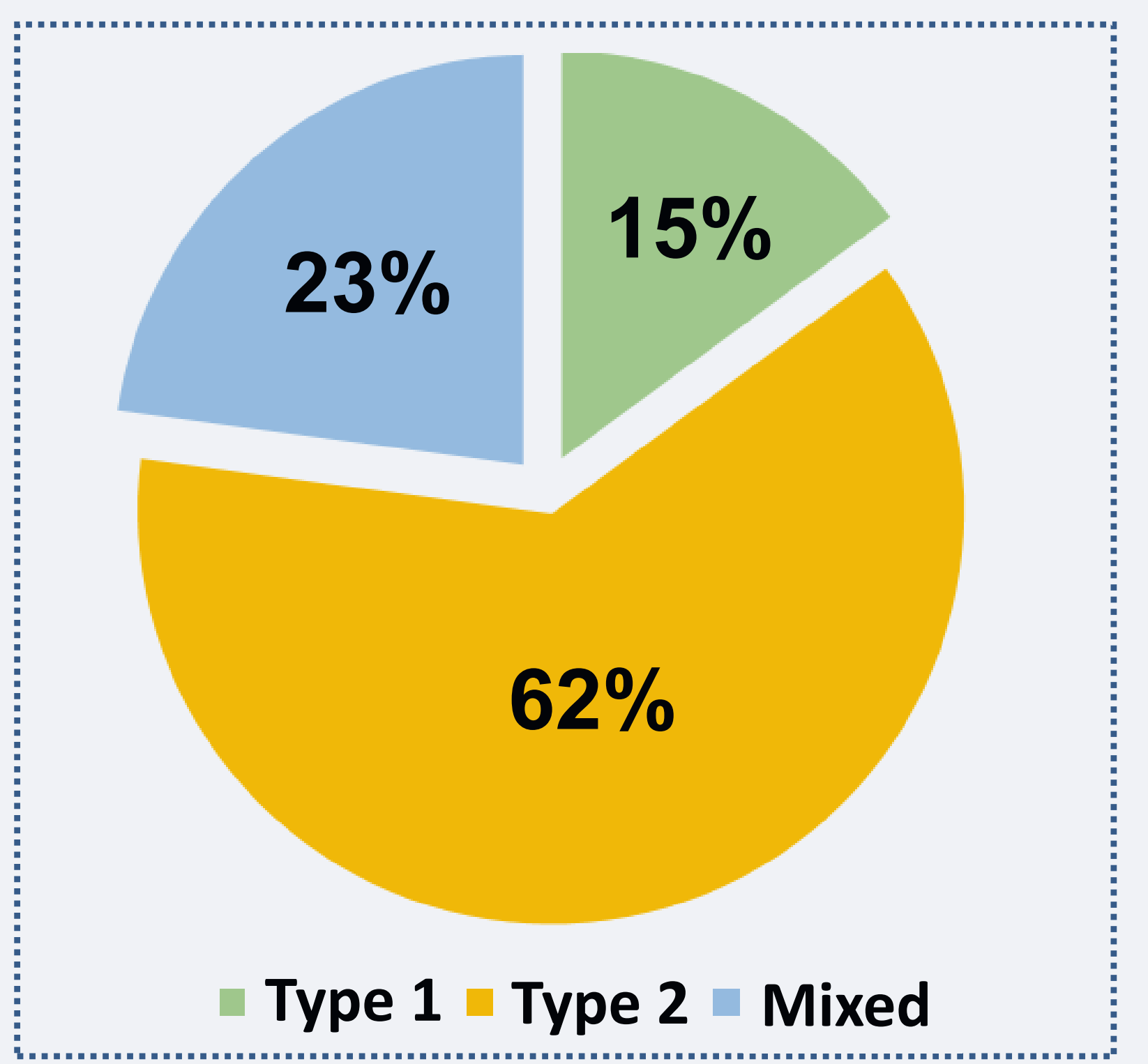

AlT Type

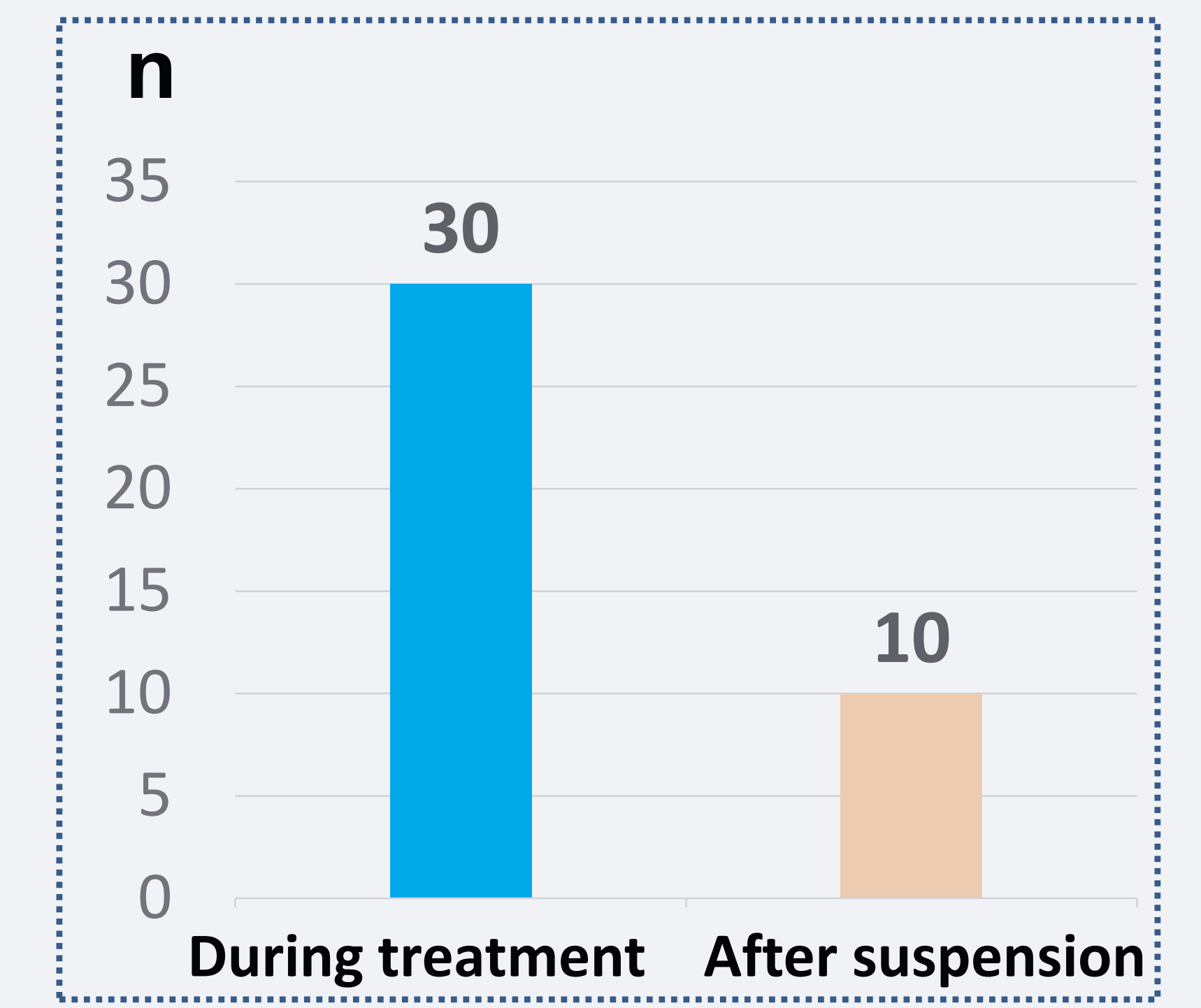

AIT development

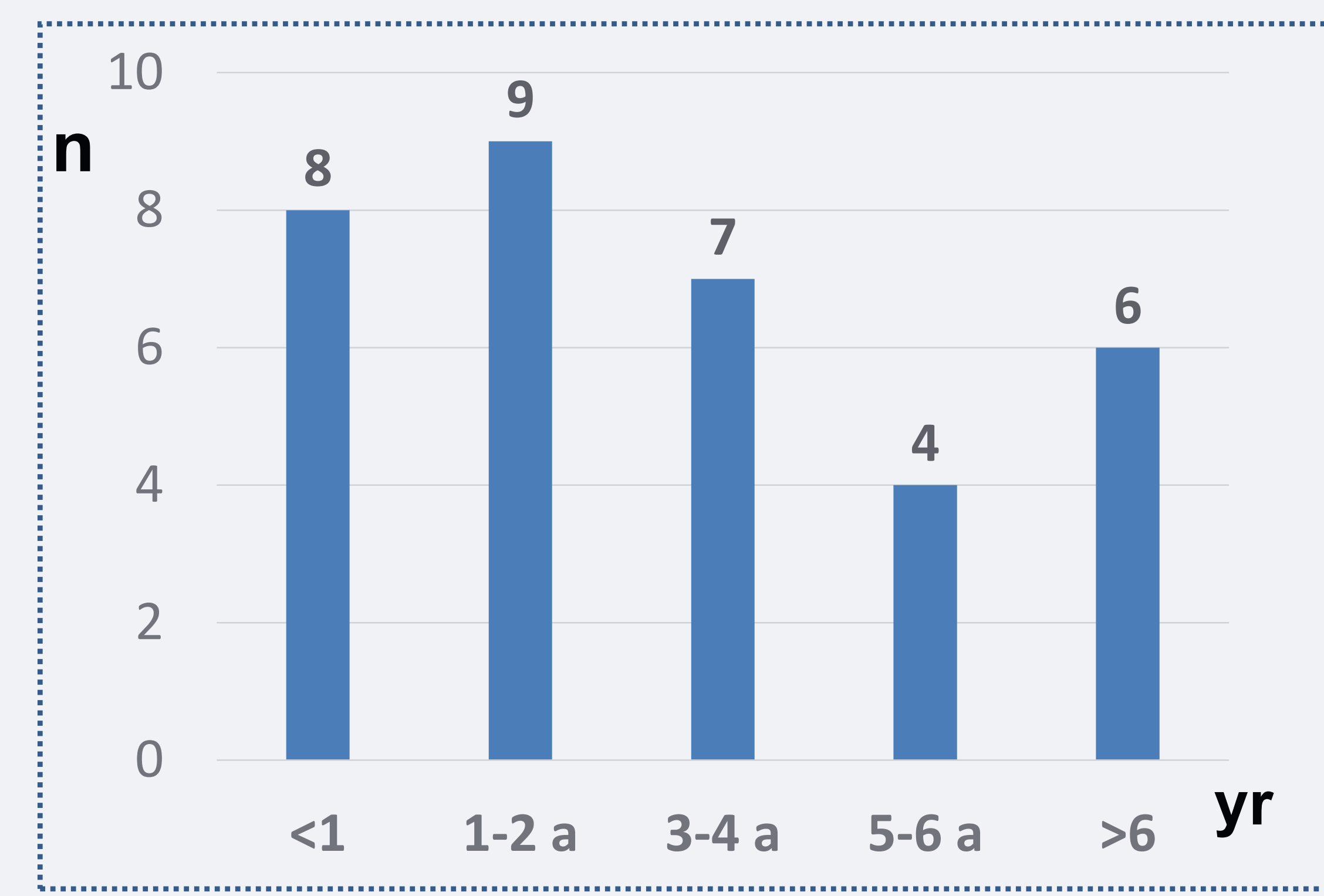

Amiodarone treatment duration

Months since suspension until AIT

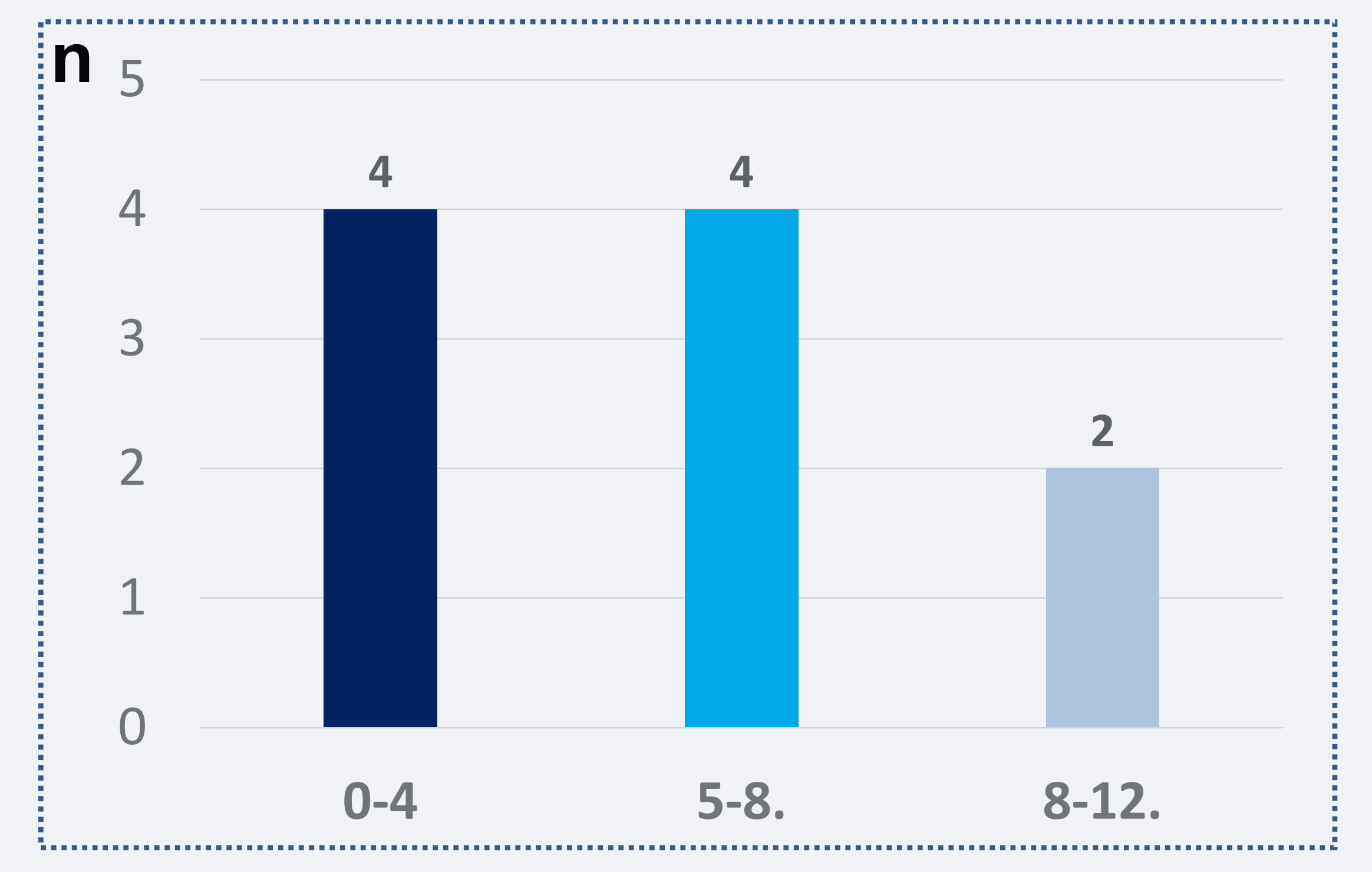

\section{Symptons and signals}

Atrial fibrillation $\quad 62,5 \%$

Cardiac insufficiency $\quad 12,5 \%$

Lethargy $4 \%$

Weight loss $\quad 4 \%$

Tremor $3 \%$

No complains $\quad 14 \%$

\section{$\underline{\text { Inpatients }}$}

$\mathbf{4 7 , 5 \%}$ due to atrial fibrillation or cardiac insufficiency

\section{Thyroid US}

Micronodular goiter 50\%

Thyroid nodule(s) $1-4 \mathrm{~cm} 23 \%$

Thyroiditis pattern $21 \%$

Normal 6\%

\section{Presentation}

Resolution
$\checkmark$ Medication 70\%
$\checkmark$ Radiodine 2,5\% (TA)
$\checkmark$ Surgery 5\% (GD)
Cardiovascular events 10
Hospitalizations 14
Mortality 3
Monitoring

Monitoring

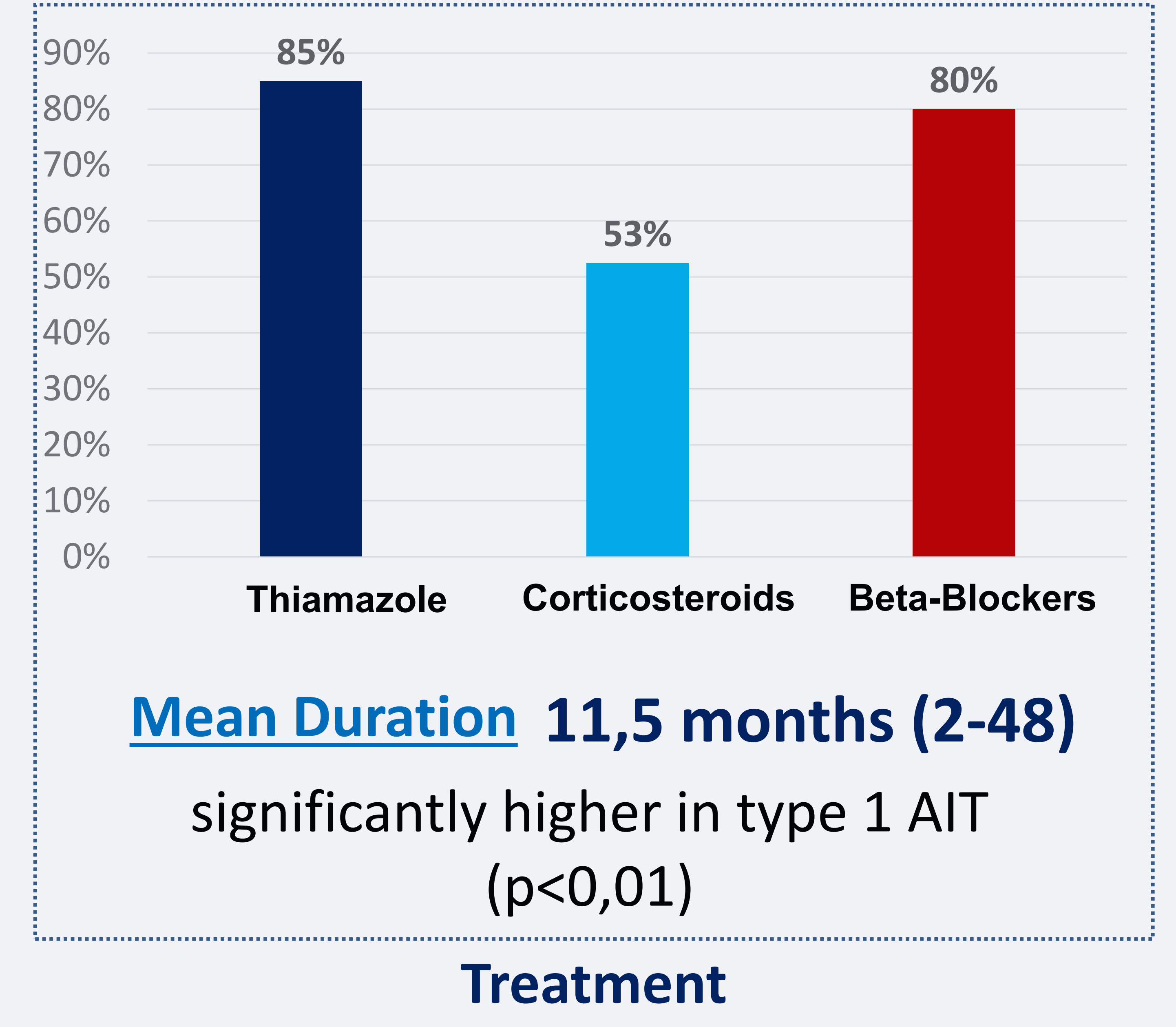

Mean Duration 11,5 months (2-48)

significantly higher in type 1 AIT $(p<0,01)$ Treatment

Workup
$(p<0.01)$
/ TRAb

\section{Conclusion}

AIT is a concern in elderly people, because treatment is challenging. Patients treated with Amiodarona and AIT need frequent hospitalizations. Type 2 AIT was the most frequent $(62,5 \%)$, with higher FT4 $(p=0,014)$, but treatment duration was smaller $(p<0,001)$. 